\title{
"INCREASED" SENSORY STIMULATION LEADS TO CHANGES IN ENERGY-RELATED ENZYMES IN THE BRAIN ${ }^{1}$
}

\author{
W. DALTON DIETRICH, ${ }^{*, 2}$ DIANNE DURHAM, ${ }^{\ddagger, 3}$ OLIVER H. LOWRY, ${ }^{*}$ AND \\ THOMAS A. WOOLSEY $\$$
} Departments of ${ }^{*}$ Pharmacology and ${ }^{\ddagger}$ Anatomy and Neurobiology and the ${ }^{\ddagger}$ McDonnell Center for Studies of Higher Brain
Function, Washington University School of Medicine, St. Louis, Missouri 63110

Received March 1, 1982; Revised June 7, 1982; Accepted June 10, 1982

\begin{abstract}
The facial whiskers of mice project through several synapses to analomically distinct structures called barrels in the contralateral cerebral cortex. With appropriate illumination, individual barrels can be recognized and dissected from unfixed, freeze-dried tissue sections taken parallel to the plane of layer IV. The tissue then can be analyzed using quantitative microhistochemical techniques to determine the level of various substances of biological importance (W. D. Dietrich, D. Durham, O. H. Lowry, and T. A. Woolsey (1981) J. Neurosci. 1: 929-935). The present paper describes results obtained in this way from adult mice subjected to a chronic "sensory deprivation" by repeatedly clipping all of the whisker hairs on one side of the face and during the recovery from this deprivation in which the whisker hairs were allowed to grow back. Sensory deprivation for 60 days leads to significant changes in the levels of the three energy-related enzymes studied-citrate synthase, malate dehydrogenase, and glycogen phosphorylase. Surprisingly, during clipping, the enzyme levels in the barrels of the contralateral cortex are essentially normal, whereas enzyme levels in the barrels of the ipsilateral cortex are increased significantly. Specifically, activities expressed as a percentage of levels in normal animals were: citrate synthase, $135 \%$; malate dehydrogenase, $130 \%$; and glycogen phosphorylase, $170 \%$. Forty-five days after the deprivation is reversed, the levels return to normal. These significant changes occurred in adult mice several synapses away from the sensory periphery. The data are in contrast to our earlier results in which damage to the primary afferents reduccd the levels of the enzymes citrate synthase and malate dehydrogenase contralateral to the manipulation. A possible explanation for the enzymatic changes observed in the cortex ipsilateral to the clipped whiskers is an increased utilization of the intact sensory periphery by the animals; this has some behavioral support.
\end{abstract}

In mice and certain other rodents, there is an "isomorphism" between the organization of the sensory and anatomically demonstrable subdivisions of the central somatosensory pathways (Woolsey et al., 1981; Labedsky and Lierse, 1968; Belford and Killackey, 1979). Fibers from the large whiskers, or mystacial vibrissae, on the face project to three centers in the ipsilateral brain-

\footnotetext{
${ }^{1}$ This work was supported by United States Public Health Service Grants NS 07057, NS 15070, NS 10244, NS 08862, and NS 07129 and Amcrican Cancer Society Grant BC4W. We wish to thank Bob Freund for photography and Jan Hoffmann for typing.

${ }^{2}$ Current address: Department of Neurology, University of Miami School of Medicine, P.O. Box 016960, Miami, FL 33101.

${ }^{3}$ Current address: Department of Otolaryngology, University of Virginia Medical Center, Box 430, Charlottesville, VA 22901.

${ }^{4}$ To whom correspondence should be addressed at Department of Anatomy and Neurobiology, Washington University School of Medicine, 660 South Euclid Avenue, St. Louis, MO 63110.
}

stem from which some cells send axons across the midline to a similar representation in the ventrobasal complex of the thalamus. Cells in the thalamus project to groups of granule cells called barrels in the somatosensory neocortex that are three synapses away from the somatosensory periphery (Woolsey and Van der Loos, 1970). The organization of the pathway can be easily demonstrated using a histochemical stain for the mitochondrial enzyme, succinic dehydrogenase (e.g., Woolsey et al., 1981; Belford and Killackey, 1979; Killackey and Belford, 1979). It is known that damage to selected groups of whiskers early in the animal's life will produce consistent and predictable changes in the anatomical organization of the cortex (Van der Loos and Woolsey, 1973; Woolsey and Wann, 1976) and the intervening substations (Belford and Killackey, 1980; Durham, 1982).

Recently, Wong-Riley and Welt (1980) showed that another mitochondrial enzyme, cytochrome oxidase, also 
demonstrated histochemically, was present at reduced levels in the relevant barrels following damage to the selected groups of whiskers in adult animals. To understand this phenomenon better, we applied our quantitative microhistochemical techniques to the cortical barrels (e.g., Lowry and Passonneau, 1972; Dietrich et al., 1981). We examined the time course of changes in the cortex produced by sensory deafferentation in the adult and used these quantitative techniques to demonstrate the extent of the changes. A necessary prerequisite was to show that the relevant structures, namely the barrels, could be identified in tissue sections which are unfixed and unstained to protect the integrity of the relevant proteins. Certain key enzymes in energy metabolism were assayed in this way-frequently, several enzymes from the same barrel-to characterize the central nervous system response to the peripheral perturbation. The principal findings of the study were that there were significant changes in the levels of the enzymes measured as a function of time after the damage to the whiskers in the adult animal and that most of the changes had approached stable levels 60 days after the initial insult (Dietrich et al., 1981).

Wong-Riley and Welt (1980) reported a second major finding: "sensory deprivation" in the adult animal results in changes in the observed intensity of cytochrome oxidase staining. An advantage of the rodent trigeminal system is that it is possible to separate a sensory deafferentation (damage to the peripheral nerves and their processes) from a sensory deprivation (removal of the whiskers alone without damage to the sensory endings). A natural extension of our quantitative histochemical studies was to examine the consequences of chronic whisker removal (a sensory deprivation) on quantitatively determined levels of various energy-related enzymes in the barrels and, further, to see whether any changes could be reversed when the whisker hairs were allowed to grow back (a reversal of the sensory deprivation). We report here the results of such an experiment and show that a chronic sensory deprivation does result in substantial changes in the levels of a number of energyrelated enzymes in the brain. However, to our surprise, the changes are not found on the side of the brain which is related to the reduced tactile input but rather on the opposite side which is related to the intact sensory periphery and which presumably receives increased natural stimulation during this experiment.

\section{Materials and Methods}

Animals and manipulations. Swiss Webster mice of both sexes were used. The animals were bred in the laboratory. All animals were at least 60 days old at the time of the initiation of the experiments. The whiskers on one side of the face were clipped with iridectomy scissors every other day for 60 days. In about half of the animals, the whisker removal was done under light barbiturate (Nembutal) anesthesia, and in about half of the animals, the procedure was done without anesthesia. Care was taken to cut the whiskers cleanly at or near the skin surface under a dissecting microscope without trauma to the hair follicles and to minimize stimulation of the whiskers during clipping. Some animals were sac- rificed after 60 days of the whisker clipping, the sensory deprivation. After the 60-day sensory deprivation, others were allowed to survive for 15 or 45 days longer without additional clipping. Within 8 to 10 days after the clipping is stopped, the whiskers approach their normal length and diameter (Ibrahim and Wright, 1975). Three or four animals were used to determine the values for each time point.

Tissue preparation. All animals were decapitated and the heads were submersed immediately in liquid nitrogen chilled to its freezing point $\left(-210^{\circ} \mathrm{C}\right)$ by partial evaporation under vacuum. The frozen brains were dissected out in a $-20^{\circ} \mathrm{C}$ cold room and divided along the midline. The hemispheres were sectioned serially at $20 \mu \mathrm{m}$ in a cryostat $\left(-20^{\circ} \mathrm{C}\right)$ in a plane parallel to layer IV of the somatosensory cortex. The sections were dried overnight at $-35^{\circ} \mathrm{C}$ under vacuum and stored at $-35^{\circ} \mathrm{C}$ until the analyses were carried out. The dried sections were viewed under a dissecting microscope on a translucent Lucite plate illuminated from below (Lowry and Passonneau, 1972). In unstained sections of this kind, it is possible to identify the barrels of layer IV readily and to dissect out individual identified barrels with special microinstruments for further analysis. Typically, three to four barrels from each hemisphere of an experimental animal were taken for analysis (see Dietrich et al., 1981, Fig. 1). In addition, as internal controls, small samples were taken from layer IV of the visual cortex to determine the levels of the enzymes citrate synthase and malate dehydrogenase in the two hemispheres of two animals after the 60day sensory deprivation.

Analytical procedures. Small tissue samples weighing as little as $20 \mathrm{ng}$ were weighed on a quartz fiber fishpole balance and analyzed as previously described (Dietrich et al., 1981). These analyses permitted the levels of the enzymes studied to be determined quantitatively.

\section{Results}

For each of the three enzymes studied in detail, we found changes in activity as a consequence of the experimental manipulation and, further, the enzymatic levels returned to normal values over time once the sensory deprivation was "reversed." This was an expected result. However, the site and the direction of the changes were quite unexpected. Namely, the enzyme levels did not change in the barrels of the cortex receiving direct inputs from the clipped whiskers but rather in the barrels of the cortex associated with the intact sensory periphery. Detailed data are shown for the mitochondrial enzyme citrate synthase in Figure 1. For comparison, we include data taken from animals in which selected whisker follicles were damaged by electrocautery 60 days prior to sacrifice (Dietrich et al., 1981). The normal levels are from nonexperimental animals. Sixty days after the whisker damage, the barrels associated with the damaged whiskers show a fall in the level of activity of this enzyme to about $70 \%$ of normal. After 60 days of whisker clipping, enzyme levels in the barrels associated with the clipped whiskers were near normal, while in contrast, the enzyme in the barrels related to the intact hairs on the opposite side showed an increase which approached $140 \%$ of normal. After the deprivation was stopped, enzyme levels in 


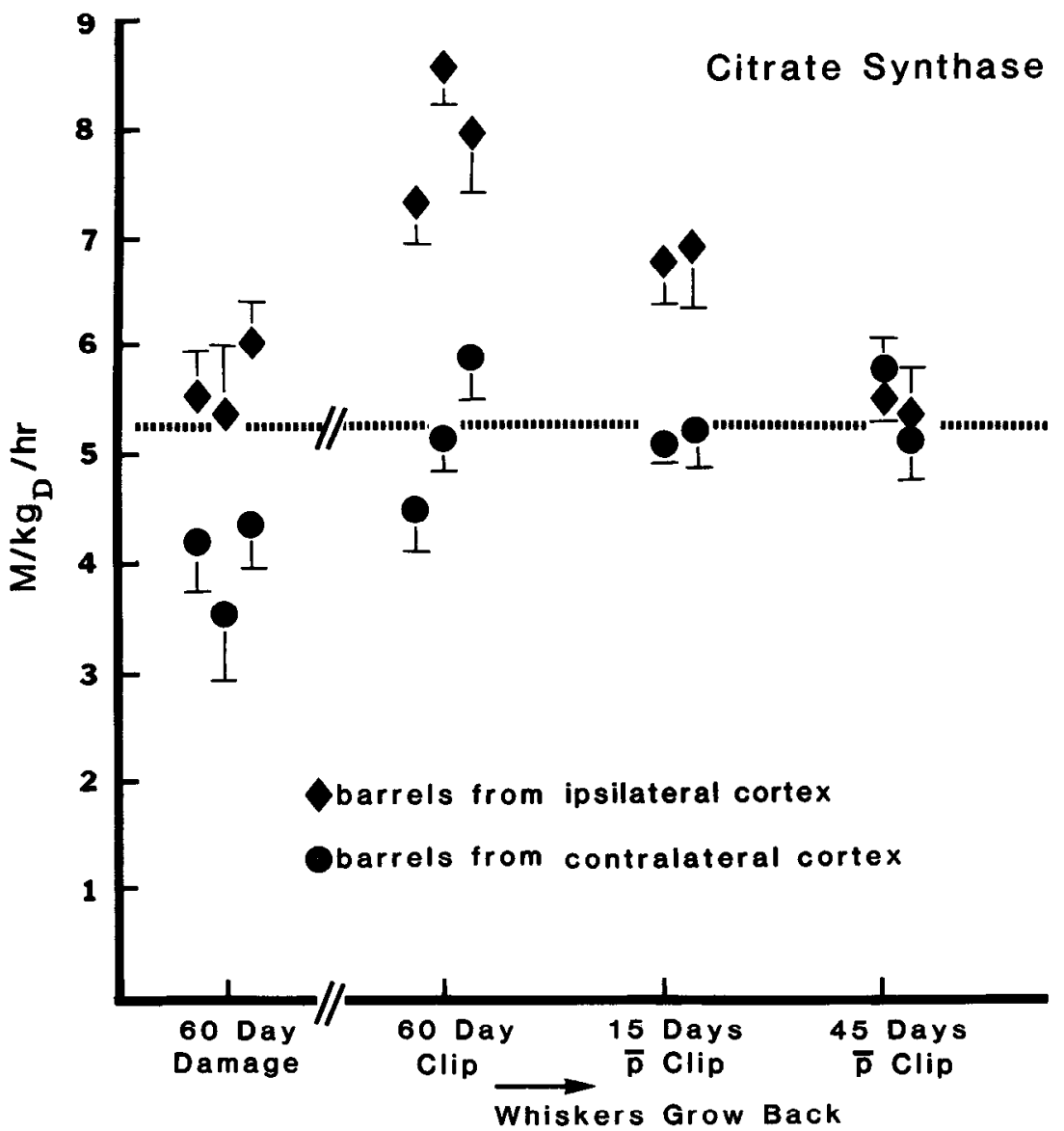

Figure 1. Activity of citrate synthase in cortical barrels 60 days after cautery of follicles to selected vibrissae or chronic clipping of the whiskers from one side of the face. The dashed line indicates the mean level of this enzyme $(5.28 \mathrm{~mol} / \mathrm{kg} \mathrm{dry} \mathrm{weight/hr)}$ determined from normal nonexperimental animals. The paired data points represent the mean value \pm SE for three to four barrels from two or three different animals. The data for the barrels related to cauterized follicles in this figure and Figure 3 are from a previous publication (Dietrich et al., 1981). After whisker cautery, changes are observed in the barrels contralateral to the manipulation, while after whisker clipping, changes are seen in the barrels ipsilateral to the manipulation. When the whiskers grow back, the levels fall to normal. $M / \mathrm{kg}_{\mathrm{D}} / \mathrm{hr}$, Moles per kg dry weight per hr.

the barrels related to the deprived side stayed near normal, while those in the barrels related to the intact side fell so that, 45 days later, levels of this enzyme were essentially normal on both sides. Trends similar to those observed for citrate synthase were seen in the data obtained for the enzymes malate dehydrogenase (mitochondria and cytosol) and glycogen phosphorylase (cytosol) (Fig. 2). Thus, there are significant differences in the nature and site of the enzymatic changes observed in the two different experiments-deafferentation and sensory deprivation.

As a simple control, we examined the levels of the enzymes citrate synthase and malate dehydrogenase in layer IV of the visual cortex of two of the animals after the 60-day whisker clipping. The levels of these enzymes, determined from four samples from each hemisphere, were virtually identical on the two sides of the brain: the values for citrate synthase were $6.40 \pm 0.40 \mathrm{~mol} / \mathrm{kg}$ dry weight $/ \mathrm{hr}$ in the right cortex and $6.57 \pm 0.62 \mathrm{~mol} / \mathrm{kg}$ dry weight/hr in the left cortex; the values for malate dehydrogenase were $25.4 \pm 1.35 \mathrm{~mol} / \mathrm{kg}$ dry weight $/ \mathrm{hr}$ on the right and $24.7 \pm 1.10 \mathrm{~mol} / \mathrm{kg}$ dry weight $/ \mathrm{hr}$ on the left. ${ }^{5}$ These findings indicate that the changes in enzyme levels that we report are probably specific for the barrel cortex and not the consequence of more global changes differentially affecting the cortex of one hemisphere.

Figure 3 compares some of the results for the three enzymes from Figure 2 with our previous results obtained after whisker damage. In the cases of citrate synthase and malate dehydrogenase, deafferentation produces a substantial reduction in the levels in the barrels associated with damaged whiskers; the levels are nearly normal in the barrels associated with clipped whiskers but are increased significantly in the barrels on the opposite side (i.e., associated with the intact whiskers). The difference between the barrels associated with the cauterized whiskers and the barrels associated with the intact whiskers

\footnotetext{
${ }^{5}$ The levels of both enzymes are 20 to $25 \%$ higher than those determined for the barrel cortex in normal control animals. Regional variations of this type are well described in the brain (e.g., Strominger and Lowry, 1955).
} 

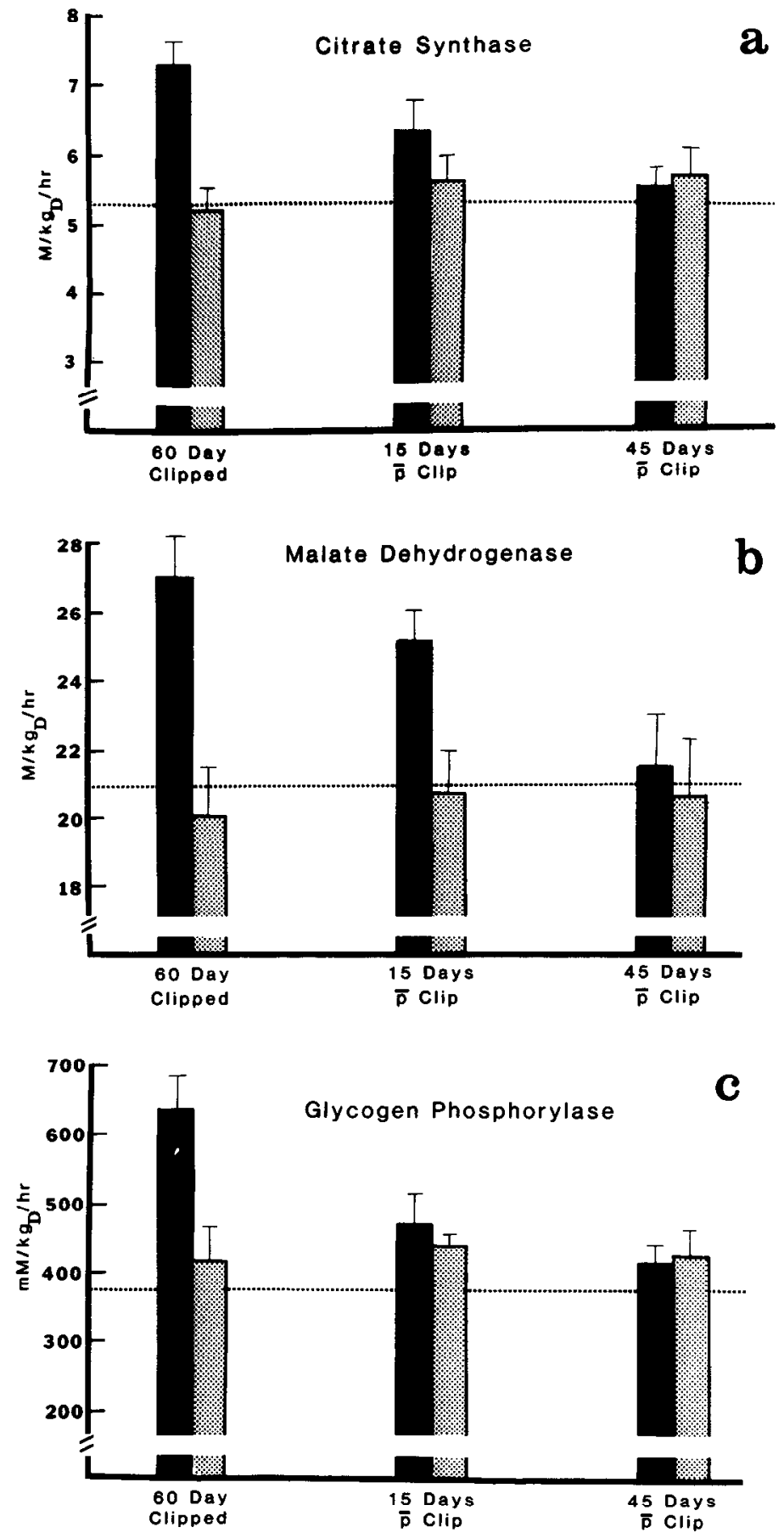

Figure 2. The levels of three enzymes in adult mouse barrels after 60 days of whisker clipping and 15 and 45 days after the whisker clipping is stopped. (The error bars indicate $\pm \mathrm{SE}$ for up to seven animals in each of which one to four barrels were analyzed, which is the reason for the differences between the values in this figure and Fig. 1.) The stippled bars indicate the levels in the barrels contralateral to the clipped whiskers. None of these enzyme levels are significantly different from those in normal animals (dashed lines). The solid bars indicate the levels in the barrels ipsilateral to the clipped whiskers (i.e., those receiving inputs from the intact whiskers). For all three enzymes, the levels are elevated significantly after 60 days of deprivation and are within normal limits within 45 days after the whiskers are allowed to grow back. $M / k^{\prime} g_{D} / h r$, Moles per $\mathrm{kg}$ dry weight per hr.

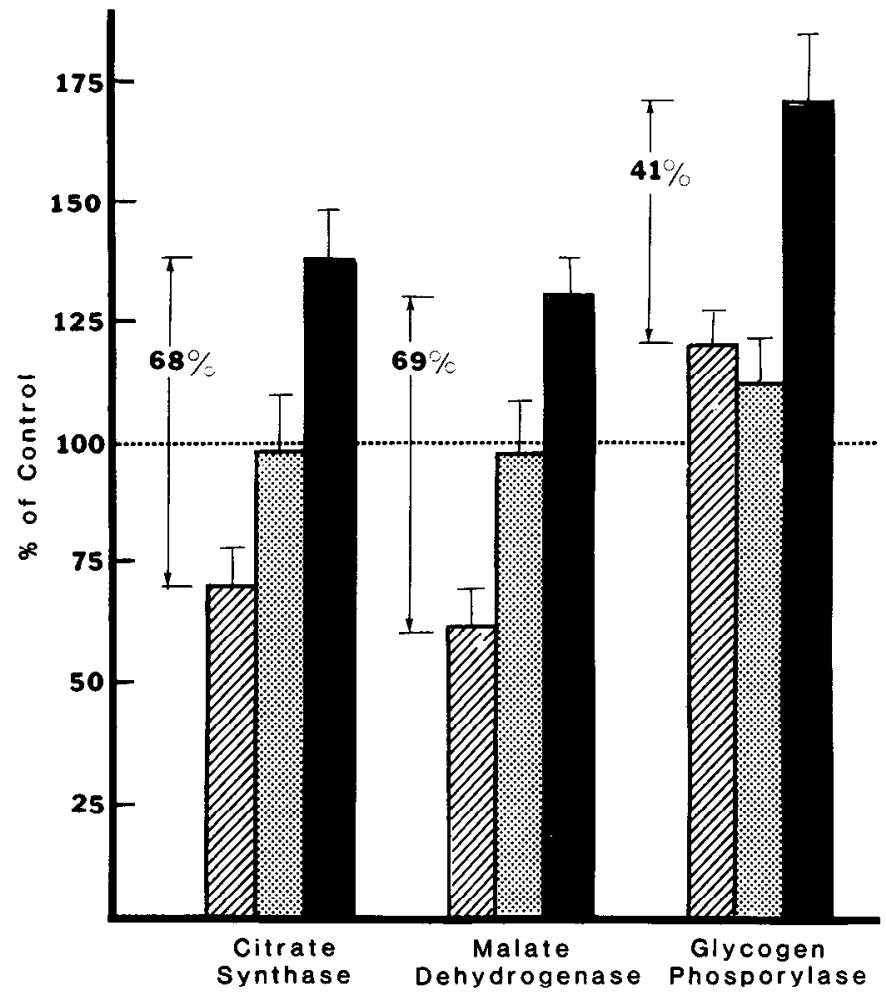

Figure 3. Normalized histograms comparing enzyme levels in the barrels associated with cauterized follicles (hatched bars), barrels associated with clipped whiskers (stippled bars), and barrels associated with the intact whiskers on the side of the face opposite to the clipped whiskers (solid bars). The error bars indicate $\pm \mathrm{SE}$ as described in the previous figure. The dashed lines indicate the levels for normal adult mice. The values were taken 60 days after the initiation of the manipulation (i.e., follicle cautery and whisker clipping). To show the relative range of the changes observed, the differences in the means (hatched versus solid bars) are expressed as a percentage of normal values.

in the clipped animals is nearly 2 -fold in the two experimental conditions. The third enzyme is of interest since the levels of glycogen phosphorylase rise in both the barrels associated with damaged whiskers and those associated with whiskers on the intact side of the deprived animals. This is in contrast to the behavior of the other two enzymes and indicates a differential response to the particular experimental manipulations employed in our studies. Nevertheless, this change, as that for the other enzymes, is reversed when the sensory deprivation is reversed.

\section{Discussion}

This study extends our findings on "plasticity" in the metabolic machinery of the nervous system. The principal findings are that the levels of certain energy-related enzymes in the adult central nervous system change in response to chronic sensory deprivation and that these changes are reversible over time when the integrity of the sensory periphery is restored. In particular, the levels of three energy-related enzymes increase in the ipsilateral cortex in response to a simple sensory deprivation of the 
opposite cortex. An interesting aspect of these results is that the changes are detected in neurons which are at least two or three synapses away from the manipulated periphery (i.e., principally, the terminals of the second order thalamocortical cells, which end in the barrels and portions of the third order of cells which comprise the barrels). No obvious anatomical change is observed in adult animals following either deafferentation or whisker clipping (Woolsey and Wann, 1976).

One interpretation of the present results is that the portions of the system associated with the intact periphery are responding to increased stimulation of that part of the body. The early findings of Vincent (1912) are of particular relevance to this interpretation. She tested the maze-running ability of normal adult rats and of adult rats in which she had clipped the whiskers on one or both sides of the face. While the rats without any vibrissae ran the maze much slower than the normal rats, the animals missing vibrissae on only one side were able to complete the maze in the same time as normal rats. Vincent (1912) noted that, whereas the normal animals oscillated from one edge of the maze to the other as if they were using the vibrissae on both sides of the face, the animals with the unilaterally clipped whiskers stayed close to the maze edge on the side of their intact whiskers as if they were using their intact periphery to run the maze. On the basis of this behavior, it might be expected that, over time, animals with unilaterally clipped whiskers would use their intact whiskers more, resulting in super normal levels of neural activity in the central pathways, including the barrels, associated with the intact whiskers. The data presented here are in many ways similar to observations on muscle enzyme changes in increased exercise (Holloszy and Booth, 1976) and in inactivity produced by immobilization (Karpati and Engel, 1968).

The present results are, as in our previous communication, phenomenological (Dietrich et al., 1981). Nevertheless, they suggest avenues by which one ultimately might determine the mechanism(s) by which these changes are provoked. The first is a comparison of the consequences of deafferentation and of increased stimulation. This comparison indicates the range of these changes and the times over which they occur (e.g., Fig. 3). Second, there are significant differences between a frank damage to the peripheral nerves, or deafferentation, and a simple sensory deprivation, suggesting that there are thresholds for the observed changes which possibly are related to levels of neuronal activity in the pathway. However, the changes are not necessarily correlated directly with energy utilization, such as demonstrated by the 2-deoxyglucose technique (e.g., Durham and Woolsey, 1978; Durham et al., 1981). It is possible that enzyme levels are not affected until neural activity falls below a threshold and that activity is not reduced to this extent by clipping alone. Third, it should be possible to dissociate the "regulation" of various enzyme levels in the pathway after different manipulations. For instance, the levels of the mitochondrially related enzymes (citratc synthase and malate dehydrogenase) are reduced following deafferentation and are increased after apparently increased stimulation, corresponding to expectations for energy utilization. However, the changes in glycogen phosphorylase levels differ in that, in both conditions, the levels of this enzyme rise. Lastly, it would be useful to compare, in greater detail, the rates of change in the levels of these and other enzymes and substrates as a consequence of the two kinds of manipulation. The data might provide insights into the mechanisms for the increase and/or decline of the synthesis of the enzymes in relation to altered CNS activity and suggest avenues for more detailed analyses designed to elucidate the regulation of these substances in the central nervous system.

The paradigm used in the present study, namely clipping all of the whiskers on one side of the face, differs from that used by Wong-Riley and Welt (1980) in which they showed a qualitative decrease in cytochrome oxidase after repeatedly clipping a single row of whisker hairs on one side of the face. In that circumstance, the levels of the enzymes that we have measured could be reduced in the deprived barrels, a problem which we are presently studying. Nevertheless, we think that it is important that we have demonstrated quantitatively an increase in the enzymes that we have studied apparently in response to increased sensory stimulation. We know of no precedent in the literature for this kind of metabolic response in the CNS.

\section{References}

Belford, G. R., and H. P. Killackey (1979) Vibrissae representation in subcortical trigeminal centers of the neonatal rat. J. Comp. Neurol. 183: 305-322.

Belford, G. R., and H. P. Killackey (1980) The sensitive period in the development of the trigeminal system of the rat. J. Comp. Neurol. 193: 335-350.

Dietrich, W. D., D. Durham, O. H. Lowry, and T. A. Woolsey (1981) Quantitative histochemical effects of whisker damage on single identified cortical barrels in the adult mouse. J. Neurosci. 1: 929-935.

Durham, D. (1982) Anatomical and functional plasticity in the developing mouse central trigeminal pathway. Doctoral dissertation, Washington University, St. Louis, MO.

Durham, D., and T. A. Woolsey (1978) Acute whisker removal reduces neuronal activity in barrels of mouse $\mathrm{SmI}$ cortex. $\mathrm{J}$. Comp. Neurol. 178: 629-644.

Durham, D., T. A. Woolsey, and L. Kruger (1981) Cellular localization of $2-\left[{ }^{3} \mathrm{H}\right]$ deoxy-D-glucose from paraffin-embedded brains. J. Neurosci. 1: 519-526.

Holloszy, J. O., and F. W. Booth (1976) Biochemical adaptations to endurance exercise in muscle. Annu. Rev. Physiol. 38: 273-291.

Ibrahim, L., and E. A. Wright (1975) The growth of rats and mice vibrissae under normal and some abnormal conditions. J. Embryol. Exp. Morphol. 33: 831-844.

Karpati, G., and W. K. Engel (1968) Correlative histochemical study of skeletal muscle after suprasegmental denervation, peripheral nerve section, and skeletal fixation. Neurology ( $N$. Y.) 18: 681-692.

Killackey, H. P., and G. R. Belford (1979) The formation of afferent patterns in the somatosensory cortex of the neonatal rat. J. Comp. Neurol. 18.3: 285-304.

Labedsky, L., and W. Lierse (1968) Die entwicklung der succinodehydrogenase-aktivität im Gehirn der maus während der postnatalzeit. Histochemie 12: 130-151.

Lowry, O. H., and J. V. Passonneau (1972) A Flexible System of Enzymatic Analysis, Academic Press, New York.

Strominger, J. L., and O. H. Lowry (1955) The quantitative 
histochemistry of brain. IV. Lactic, malic and glutamic dehydrogenases. J. Biol. Chem. 213: 635-646.

Van der Loos, H., and T. A. Woolsey (1973) Somatosensory cortex: Structural alterations following early injury to sense organs. Science 179: 395-398.

Vincent, S. B. (1912) The function of the vibrissae in the behavior of the white rat. Behav. Monogr. 1: 1-81.

Wong-Riley, M. T. T., and C. Welt (1980) Histochemical changes in cytochrome oxidase of cortical barrels after vibrissal removal in neonatal and adult mice. Proc. Natl. Acad. Sci. U. S. A. 77: 2333-2337.
Woolsey, T. A., and H. Van der Loos (1970) 'The structural organization of layer IV in the somatosensory region (SI) of mouse cerebral cortex. Brain Res. 17: 205-242.

Woolsey, T. A., and J. R. Wann (1976) Areal changes in mouse cortical barrels following vibrissal damage at different postnatal ages. J. Comp. Neurol. 170: 53-66.

Woolsey, T. A., D. Durham, R. M. Harris, D. J. Simons, and K. L. Valentino (1981) Somatosensory development. In Development of Perception, R. N. Aslin, J. R. Alberts, and M. R. Petersen, eds., Vol. 1, pp. 259-292, Academic Press, New York. 